Gómez-López, M.; Manzano-Sánchez, D.; Merino-Barrero, J.A. y Valero-Valenzuela, A. (2020) Causes of Success in Handball through the Beliefs about Ability. Revista Internacional de Medicina y Ciencias de la Actividad Física y el Deporte vol. 20 (77) pp. 103-118 Http://cdeporte.rediris.es/revista/revista77/artcreencias1126.htm

DOI: $10.15366 /$ rimcafd2020.77.007

\title{
ORIGINAL
}

\section{CAUSAS DEL ÉXITO EN BALONMANO A TRAVÉS DE LAS CREENCIAS SOBRE HABILIDAD}

\section{CAUSES OF SUCCESS IN HANDBALL THROUGH THE BELIEFS ABOUT ABILITY}

\author{
Gómez-López, M.'; Manzano-Sánchez, D. ${ }^{1}$; Merino-Barrero, J.A. ${ }^{2}$ y Valero- \\ Valenzuela, A. $^{1}$ \\ ${ }^{1}$ Departamento de Actividad Física y Deporte. Universidad de Murcia (España) mgomezlop@um.es, \\ david.manzano@um.es, avalero@um.es \\ 2 Facultad de Educación. Universidad Internacional de La Rioja (UNIR), Logroño (España) \\ jandres.merino@unir.net
}

\section{Agradecimientos}

Este trabajo ha sido realizado gracias al apoyo y la colaboración de la Real Federación Española de Balonmano (RFEBM) y de la Federación Andaluza de Balonmano (FABM) que facilitaron el trabajo de campo.

Código UNESCO / UNESCO code: 6106.08 Motivación / Motivation; 6102.01 Psicología del deporte/Sport psychology

Clasificación Consejo de Europa / Council of Europe classification: 15. Psicología del deporte / Sport psychology

Recibido 27 de abril de 2017 Received April 27, 2017

Aceptado 6 de noviembre de 2018 Accepted November 6, 2018

\section{RESUMEN}

El objetivo fue determinar la capacidad de predicción de las creencias implícitas de la habilidad sobre las creencias de las causas del éxito en el deporte. La muestra estuvo compuesta por 444 deportistas de alto rendimiento (233 chicos y 211 chicas; $\mathrm{M}=16,70 ; \mathrm{DT}=0,49$ ). Estos deportistas cumplimentaron el Inventario de Percepción de las Creencias sobre las Causas del Éxito en el deporte y la Escala de Creencias Implícitas sobre la Habilidad. Los resultados reflejaron que las 
creencias de habilidad incremental predicen el esfuerzo, mientras que las creencias de habilidad como entidad predicen la capacidad y el engaño como causas del éxito en balonmano.

PALABRAS CLAVE: entrenador, creencia incremental, creencia de entidad, engaño

\section{ABSTRACT}

The main of this study was to determine the prediction of ability implicit beliefs of sport success causes in sport. The sample was comprised of 444 youth high performance players (233 boys and 211 girls; $M=16,70 ; S D=0,49$ ). These players completed the Spanish version of the Beliefs about the Causes of Success in Sport Questionnaire and the Conceptions of the Nature of Athletic Ability Questionnaire-2. The results revealed that belief of incremental ability predict effort, while ability beliefs as an entity predict the capability and the deception as the causes of success in handball.

KEYWORDS: coach, incremental belief, entity belief, scam

\section{INTRODUCCIÓN}

Investigaciones recientes demuestran que el contexto deportivo es un lugar idóneo para lograr consecuencias positivas a través de la actividad física (Jõesaar \& Hein, 2011). Es fundamental para lograr estos beneficios tener en cuenta los aspectos motivacionales, los cuales, son relevantes para entender la conducta de los seres humanos (King \& Mclnerney, 2012).

Una de las teorías que explica la motivación, es la teoría de las metas de logro (Nicholls, 1989), la cual establece que los deportistas actúan movidos por la necesidad de mostrar su competencia y evitar demostrar lo contrario. En deportes de equipo con deportistas de alto nivel, aquellos que afirman poseer una alta orientación hacia la tarea logran una mejor competencia así como niveles de motivación más autodeterminados (Fernández, Cecchini, \& Méndez, 2017).

Centrándonos en el balonmano, son diversos los estudios recientes que se han llevado a cabo relacionando diversas variables psicológicas con la motivación (Gómez-López, Granero-Gallegos, Baena-Extremera, \& Abraldes, 2014; RuizSánchez, Gómez-López, \& Granero-Gallegos, 2017; Ruiz-Sánchez, Gómez-López, Granero-Gallegos, \& González-Hernández, 2017). Investigaciones como la de Li et al. (2011), indican que aquellos deportistas con mayores valores de orientación hacia la tarea, son también los que tienen mayor nivel competitivo y de motivación intrínseca así como un mayor disfrute, al contrario que el grupo con un mayor grado de implicación hacia el ego. 
La literatura ha evidenciado que las orientaciones de meta son buenas predictoras de las creencias sobre las causas de éxito en el deporte, tanto en deportes colectivos (Usán, Salavera, Murillo, \& Álvarez-Medina, 2017; White, Kavussanu, Tank, \& Wingate, 2004), como individuales (Abraldes, Gómez-López, GraneroGallegos, \& Rodríguez-Suárez, 2013; Abraldes, Granero-Gallegos, BaenaExtremera, Gómez-López, \& Rodríguez-Suárez, 2016; Mascret, Falconetti, \& Cury, 2016; Ruiz-Juan, Gómez-López, Pappous, Alacid, \& Flores, 2010), estando la orientación hacia el ego más relacionada con la percepción de las causas del éxito en el deporte a través del uso del engaño. Por el contario, la orientación hacia la tarea se relaciona con el esfuerzo y la capacidad personal como principales causas del éxito deportivo. Más concretamente en balonmano, estudios como el de Granero et al. (2017) indican que una orientación hacia la tarea, se relaciona con la creencia en el esfuerzo como medio para lograr el éxito deportivo, mientras que un clima orientado al ego tiene mayor relación con las creencias en la capacidad y el engaño.

Otro factor importante relacionado con la motivación de los adolescentes en el ámbito deportivo son las creencias implícitas sobre la habilidad deportiva (Li \& Lee, 2004), las cuales ejercen una fuerte influencia sobre el comportamiento. Los deportistas pueden concebir la habilidad como algo mejorable por medio del aprendizaje, esfuerzo y entrenamiento (creencia incremental), o como algo innato y estable, y por lo tanto independiente tanto de la práctica como del esfuerzo (creencia entidad) (Biddle, Wang, Chatzisarantis, \& Spray, 2003). Spray, Wang, Biddle, Chatzisarantis y Warbuton (2006) afirman que la habilidad está influida por un talento natural innato que puede ser modificada a través del esfuerzo y el entrenamiento.

En este sentido, diferentes estudios muestran que la creencia incremental de habilidad se relaciona positivamente con las formas de motivación más autodeterminadas, la diversión, la persistencia y las orientaciones de meta a la maestría, mientras que los deportistas con una creencia de la habilidad estable experimentan sentimientos de frustración y desmotivación en el momento de no alcanzar resultados satisfactorios (Li, Lee, \& Solmon, 2005).

Por tanto, son numerosos los trabajos que se han llevado a cabo relacionando el clima motivacional con las creencias del éxito deportivo (Abraldes et al., 2016; Cordo-Cabal, Gómez-López, \& Granero-Gallegos, 2019; Laparidis, Papaioannou, Vretakou, \& Morou, 2003; Mascret, Falconetti, \& Cury, 2016; Usán et al., 2017), o bien el clima motivacional y las creencias implícitas sobre la habilidad deportiva (Cury et al., 2002; González-Cutre et al., 2007; Li et al., 2005; Ommundsen, 2001).

En esta línea, Nicholls (1992) considera que las concepciones o creencias implícitas de habilidad se relacionan con la comprensión por parte de los sujetos de las diferencias entre capacidad y esfuerzo (creencias de las causas de éxito), considerando que una concepción estable de habilidad representa la creencia de que la habilidad no puede ser modificada con el esfuerzo, y la actuación depende 
de la capacidad, mientras que una concepción de habilidad como adquirida, implica que puede ser mejorada con el esfuerzo.

Por lo tanto y como ha quedado reflejado hasta el momento, las investigaciones se han centrado en relacionar por un lado el clima motivacional generado por el entrenador normalmente y las creencias de las causas del éxito en el deporte y por otro lado, este clima motivacional y las creencias implícitas de la habilidad deportiva, sin embargo, hasta la fecha no se han encontrado estudios que hayan analizado la relación predictiva entre las creencias de éxito en el deporte y las creencias implícitas de habilidad deportiva.

Por esta razón, este estudio tiene como objetivo analizar la relación predictiva de las creencias implícitas de habilidad sobre las causas del éxito en el deporte. En relación a ello, se hipotetizó que: a) la creencia implícita incremental de habilidad basada en la mejora predecirá positivamente el esfuerzo y negativamente la capacidad y el engaño como principales causas del éxito en el deporte; b) la creencia implícita incremental de habilidad basada en el aprendizaje predecirá positivamente las creencias de las causas del éxito deportivo basadas en el esfuerzo y en la capacidad y negativamente la basada en el engaño; c) la creencia implícita de entidad basada en el talento predecirá positivamente la creencia de la causa del éxito basada en la capacidad y en el engaño y negativamente en el esfuerzo; d) la creencia implícita de entidad estable predecirá positivamente las creencias del éxito deportivo basadas en la capacidad, el engaño y el esfuerzo (ver Figura 1). 


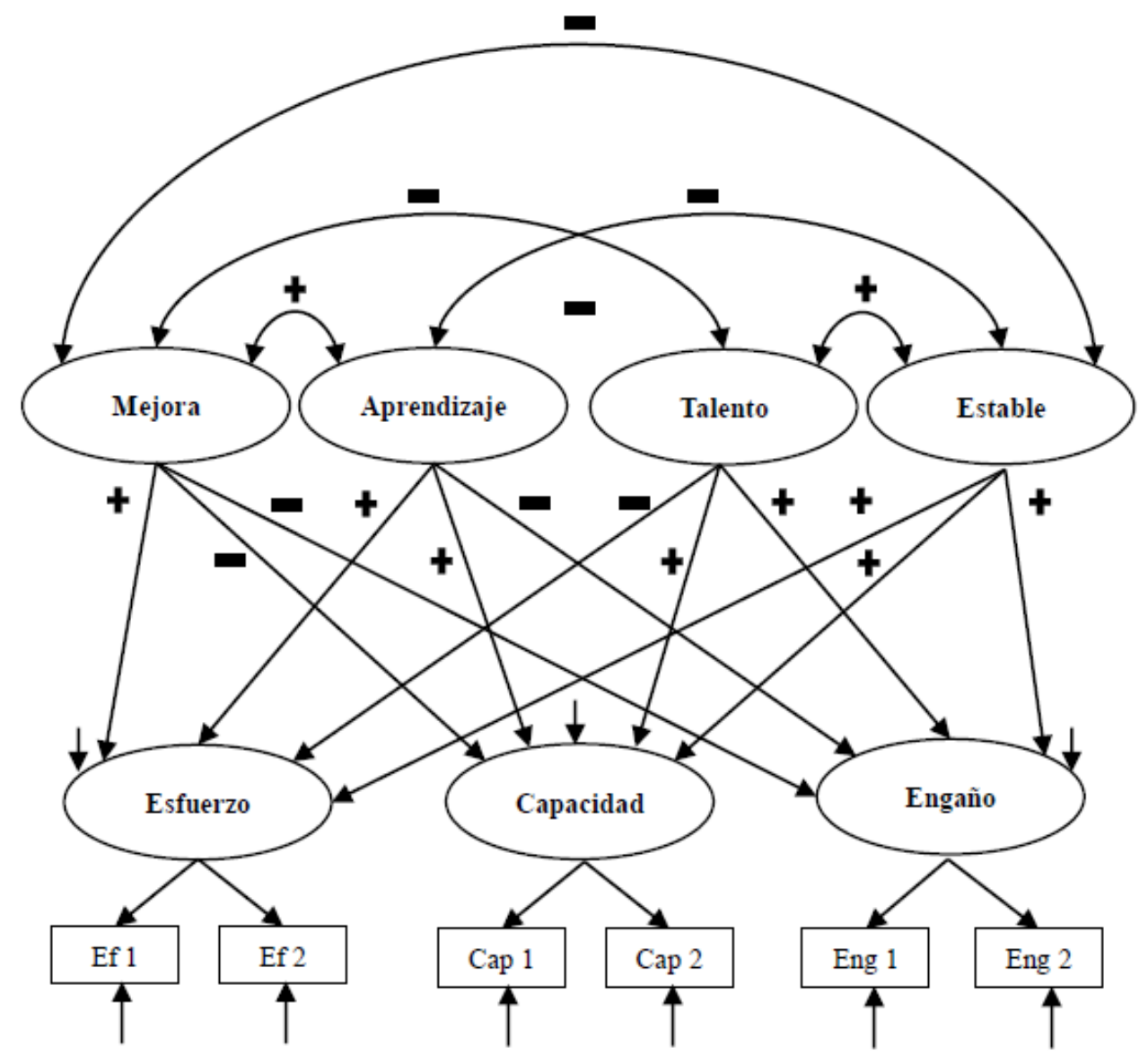

Figura 1. Modelo estructural hipotetizado sobre las relaciones que se esperan entre las variables de estudio.

\section{MATERIAL Y MÉTODOS}

\subsection{Muestra}

En el estudio participaron 444 jugadores de balonmano pertenecientes a la totalidad de selecciones que disputaron el pasado Campeonato de España de Selecciones Autonómicas (CESA) en la categoría juvenil (233 chicos y 211 chicas; $M=16,70$; DT $=0,49$ ). Estos jugadores están catalogados como deportistas de Alto Rendimiento según el Real Decreto 971/2007, de 13 de julio, sobre deportistas de Alto Nivel y Alto Rendimiento.

\subsection{Instrumentos}

Inventario de Percepción de las Creencias sobre las Causas del Éxito en el Deporte (Beliefs about the Causes of Success in Sport Questionnaire, BACSSQ; Duda, Fox, Biddle, \& Armstrong, 1992; Duda \& Nicholls, 1992). Se utilizó la versión española del inventario (Castillo, Balaguer, \& Duda, 2002), compuesto por 18 ítems que miden las percepciones que tienen los deportistas sobre si el esfuerzo (nueve ítems), la 
capacidad (cuatro ítems) y el uso de técnicas de engaño (cinco ítems) permiten alcanzar el éxito en el deporte. En las instrucciones del inventario se les pregunta a los deportistas: "¿Qué crees que debería hacer la gente para tener éxito en el deporte que practica más a menudo?', debiendo responder en escala tipo Likert de cinco puntos, desde (1) muy en desacuerdo a (5) muy de acuerdo. Estudios previos han mostrado su fiabilidad en el ámbito de la actividad físico-deportiva (Abraldes et al., 2016; Cervelló, Escartí, \& Balagué, 1999; Guivernau \& Duda, 1994; Ruiz-Juan et al., 2010) con valores $\alpha=0,74$ a $\alpha=0,87$ (esfuerzo), $\alpha=0,62$ a $\alpha=0,81$ (capacidad) y $\alpha=0,71$ a $\alpha=0,72$ (técnicas de engaño). En el presente estudio la consistencia interna de las diferentes subescalas fueron $\alpha=0,77$ (esfuerzo), $\alpha=$ 0,73 (capacidad) y $\alpha=0,84$ (técnicas de engaño). La escala total obtuvo un valor $\alpha$ $=0,79$.

Escala de Creencias Implícitas sobre la Habilidad (Conceptions of the Nature of Athletic Ability Questionnaire-2, CNAAQ-2; Biddle et al., 2003). Se utilizó la versión española de la escala (Moreno, Cervelló, Martínez, \& Moreno, 2013), compuesta por 12 ítems divididos en dos subescalas de orden superior denominadas creencia incremental y creencia de entidad. La subescala de creencias de entidad está compuesta por seis ítems de los cuales tres corresponden a la variable estable de primer orden y los otros a la variable talento. Por su parte, la subescala incremental está formada por seis ítems de los cuales tres corresponden a la variable mejora de primer orden y los otros a la variable aprendizaje. En las instrucciones de la escala se les pregunta a los deportistas: "Tus creencias sobre tú habilidad en deporte son...", debiendo responder en escala tipo Likert de cinco puntos, desde (1) totalmente en desacuerdo a (5) totalmente de acuerdo. Tanto en el estudio de Moreno et al. (2013) como en el presente, se obtuvieron valores de $\alpha=0,72$ (entidad-estable), $\alpha=0,86$ (entidad-talento), $\alpha=0,73$ (incremental-aprendizaje) y $\alpha$ $=0,68$ (incremental-mejora). Por otro lado, en el reciente estudio de Sáenz-López, Mateos, Almagro y Conde (2017) se obtuvieron valores de $\alpha=0,67$ (entidad) y $\alpha=$ 0,83 (incremental).

\subsection{Procedimiento}

Se pidió permiso a la Real Federación Española de Balonmano (RFEBM), a la Federación Andaluza de Balonmano (FABM) como sede organizadora, así como a las distintas selecciones juveniles participantes, mediante una carta en la cual se adjuntaba un ejemplar del instrumento y se explicaba los objetivos de la investigación y cómo se iba a realizar. La recogida de los datos se realizó de manera auto-administrada, en los distintos hoteles en los cuales se hospedaban las selecciones participantes durante los momentos de descanso de los jugadores y con el consentimiento previo de los jugadores, entrenadores responsables de los equipos y tutores legales. Los participantes fueron informados del objetivo del estudio, voluntariedad, absoluta confidencialidad tanto de las respuestas como del tratamiento de los así como de la no existencia de respuestas correctas o incorrectas. Asimismo, se les solicitó que contestaran las preguntas con la máxima sinceridad y honestidad. La duración aproximada de la administración del 
instrumento fue de 30 minutos de promedio. Se respetaron en todo momento todos los procedimientos éticos en la recogida de los datos y se obtuvo la aprobación del Comité de Ética de la Universidad de Murcia.

\subsection{Análisis de los datos}

Se comprobó la existencia de casos atípicos, los cuales fueron eliminados del estudio teniendo en cuenta los valores extremos mediante la distancia de Mahalanobis además de la detección de outliers, considerando una puntuación típica > 3 como distribución inadecuada como valor absoluto. Se calcularon los estadísticos descriptivos de las diferentes variables de estudio así como las correlaciones bivariadas. Posteriormente, se realizó un análisis de regresión estructural con el objetivo de testar las relaciones hipotetizadas entre las variables del estudio (ver Figura 1). Para ello, se llevó a cabo una aproximación por pasos, tal y como recomiendan Anderson y Gerbing (1988), realizando en primer lugar un modelo de medida que permita dar validez de constructo a los instrumentos $y$, seguidamente, un modelo de predicción de las variables en las que se analiza las influencias de unas variables sobre otras contempladas en el modelo planteado.

Se realizó igualmente un análisis de normalidad de cada una de las variables para determinar el uso de pruebas paramétricas o no paramétricas en función de la normalidad hallada. Los contrastes de normalidad realizados sobre la homogeneidad de la varianza (Estadístico de Levene, $p>0,05$ ) aceptaron la hipótesis de normalidad para la mayoría de las variables, lo que motivó el uso de procedimientos paramétricos. Todos los análisis se llevaron a cabo con el paquete estadístico SPSS 19.0 y Amos 19.0.

\section{RESULTADOS}

\subsection{Análisis descriptivo y correlaciones bivariadas}

Se presentan los estadísticos descriptivos (media, desviación típica, asimetría y curtosis), los valores alfa de Cronbach para cada una de las subescalas así como las correlaciones bivariadas para todas las variables objeto de estudio (ver Tabla 1). Los datos indicaron una mayor puntuación en las creencias sobre las causas del éxito hacia el esfuerzo y en las creencias implícitas sobre la habilidad, incrementalaprendizaje e incremental-mejora ( $M=4,54 ; 4,32 ; 4,37$ respectivamente), en contraste con la creencia en el engaño y la percepción de habilidad como entidadestable $(M=1,99 ; 2,21$ respectivamente $)$.

El análisis de correlaciones bivariadas reflejó una relación significativa entre las variables, excluyendo las creencias de habilidad como incremental en ambas dimensiones y la creencia en la capacidad como causa del éxito deportivo. De este modo, las subescalas relativas a la creencia incremental de habilidad mantuvieron correlación negativa con la creencia estable de habilidad pero se asociaron positivamente con la causa de éxito basado en el esfuerzo y negativamente con las 
basadas en el engaño. Por el contrario, las subescalas referentes a la creencia estable de habilidad correlacionaron negativamente con las relativas a creencia incremental y con causas de éxito basado en el esfuerzo. De otro modo, fue positiva su relación con las causas de éxito basada en la capacidad y en el engaño.

Los índices de asimetría y curtosis obtuvieron valores inferiores a 2, lo que indica normalidad univariada de los datos (Bolleng \& Long, 1993).

Tabla 1. Estadísticos descriptivos y correlaciones bivariadas de la muestra

\begin{tabular}{llccccccccccccc}
\hline & Rango & $M$ & $D T$ & $A$ & $C$ & $\alpha$ & 1 & 2 & 3 & 4 & 5 & 6 & 7 \\
\cline { 2 - 11 } & E-Estable & $1-5$ & 2,21 & 0,81 & 0,40 & $-0,18$ & 0,72 & - & $0,33^{*}$ & $-0,25^{* *}$ & $-0,33^{* *}$ & $-0,13^{* *}$ & $0,14^{* *}$ & $0,24^{* *}$ \\
2 & E-Talento & $1-5$ & 2,70 & 1,03 & 0,06 & $-0,67$ & 0,86 & - & - & $-0,19^{* *}$ & $-0,15^{* *}$ & $-0,16^{* *}$ & $0,27^{* *}$ & $0,25^{* *}$ \\
3 & I-Aprendizaje & $1-5$ & 4,32 & 0,67 & $-0,99$ & 0,37 & 0,73 & - & - & - & $0,60^{* *}$ & $0,34^{* *}$ & $-0,02$ & $-0,29^{* *}$ \\
4 & I-Mejora & $1-5$ & 4,37 & 0,64 & $-0,98$ & 0,46 & 0,68 & - & - & - & - & $0,35^{* *}$ & 0,00 & $-0,25^{* *}$ \\
5 & Esfuerzo & $1-5$ & 4,54 & 0,50 & $-1,14$ & 0,81 & 0,84 & - & - & - & - & - & $0,16^{* *}$ & $-0,21^{* *}$ \\
6 & Capacidad & $1-5$ & 3,42 & 0,91 & $-0,10$ & $-0,55$ & 0,73 & - & - & - & - & - & - & $0,34^{* *}$ \\
7 Engaño & $1-5$ & 1,99 & 0,84 & 0,72 & $-0,16$ & 0,77 & - & - & - & - & - & - & - \\
\hline
\end{tabular}

\subsection{Modelo de medición}

En primer lugar, se realizó la validación del modelo de medición planteando un análisis en el que las variables latentes correlacionaron libremente, parcelando los ítems de cada variable por pares, de modo que la mitad de los primeros ítems de cada sub-escala fueron promediados para formar el primer bloque de ítems y la segunda mitad de ítems fueron promediados para formar el segundo bloque de ítems. Marsh (1994) propuso el uso de los pares de ítems porque los resultados de éstos son más fiables, tienden a ser distribuidos de forma más normal al reducir el número de variables observadas, quedando el modelo identificado al estar medido cada variable latente por al menos dos indicadores (McDonald \& Ho, 2002).

Se comprobó la normalidad multivariada mediante el coeficiente de Mardia $(13,54)$, considerándose como adecuado dado que valores inferiores a 70 en este índice indican que el alejamiento de la normalidad multivariante no es inconveniente para el análisis (Rodríguez-Ayán \& Ruiz, 2008). Por otro lado, se cumplió el supuesto de multicolinealidad al ser las correlaciones bivariadas entre las variables inferiores a 0,85 (Pérez, Medrano, \& Sánchez Rosas, 2013). Los errores de las variables endógenas eran independientes al no estar correlacionados con otras variables. Se utilizó el método de máxima verosimilitud como método de estimación.

Teniendo en cuenta que se desaconseja utilizar una única medida de ajuste global del modelo, se calcularon diferentes índices de ajuste absolutos y relativos (Bentler, 2007; Markland, 2007; Miles \& Shevlin, 2007). Como índices absolutos se utilizó el $\mathrm{X}^{2}$ así como la ratio entre el índice $\mathrm{X}^{2} /$ g.l. (grados de libertad del modelo). Como 
relativos, se calculó el índice IFI, CFI y TLI. También se analizó en índice RMSEA y RMSR (Kline, 2005). Los índices de bondad de ajuste que se obtuvieron en el modelo de mediciones fueron adecuados con un $\mathrm{X}^{2}(18, \mathrm{~N}=444)=24,66 ; p<0,008$; $\mathrm{X}^{2}$ / g.I. = 1,98; $\mathrm{CFI}=0,98 ; \mathrm{IFI}=0,98 . \mathrm{TLI}=0,95 ; \mathrm{RMSEA}=0,04 ; \mathrm{RMSR}=0,03$.

\subsection{Modelo de regresión estructural}

Con el objeto de analizar las relaciones existentes en las variables pertenecientes al modelo que se plantea, se hipotetizó que las creencias implícitas de habilidad predicen las creencias sobre las causas del éxito deportivo, y para ello se realizó un modelo de regresión estructural. Se empleó el coeficiente de Mardia $(13,54)$ y la matriz de covarianzas como entrada para el análisis de los datos. Los índices de bondad de ajuste mostraron valores adecuados para los datos (Hu \& Bentler, 1999), ajustándose a los parámetros establecidos: $X^{2}(16, N=444)=27,75 ; p<0,01 ; X^{2}$ / g.l. = 1,95; $\mathrm{CFI}=0,98 ; \mathrm{NFI}=0,96 ; \mathrm{RMSEA}=0,03 ; \mathrm{SRMR}=0,03$. La contribución de cada uno de los factores a la predicción de otras variables se examinó a través de los pesos de regresión estandarizados. El valor $t$ asociado a cada peso se tomó como una medida de la contribución, de modo que valores superiores a 1,96 se consideran como significativos (Hair, Black, Balbin, \& Anderson, 2009). Todas las relaciones expuestas (ver Figura 2) fueron significativas. Los resultados reflejaron una predicción positiva de la creencia de habilidad incremental-mejora sobre el esfuerzo $(\beta=0,20)$ e inversamente sobre el engaño $(\beta=-0,22)$. Por otro lado, la creencia implícita de habilidad incremental-aprendizaje predijo positivamente la creencia del éxito basada en el esfuerzo $(\beta=0,26)$ y en la capacidad $(\beta=0,09)$ y negativamente la creencia basada en el engaño $(\beta=-0,09)$.

En cuanto a las creencias implícitas de habilidad como entidad-talento predijo positivamente las creencias sobre las causas del éxito deportivo basadas en la capacidad $(\beta=0,31)$ y en el engaño $(\beta=0,20)$ y negativamente en el esfuerzo $(\beta=$ $-0,10)$. Por otro lado, la creencia implícita de habilidad como entidad-estable predijo la capacidad $(\beta=0,09)$, el engaño $(\beta=0,14)$ y el esfuerzo $(\beta=0,02)$ como creencias de las causas del éxito en el balonmano.

Las relaciones analizadas explicaron el $19 \%$ de la varianza de la creencia de la causa del éxito en el balonmano basada en el esfuerzo, el $12 \%$ de la basada en la capacidad y el $21 \%$ de la basada en el engaño. 


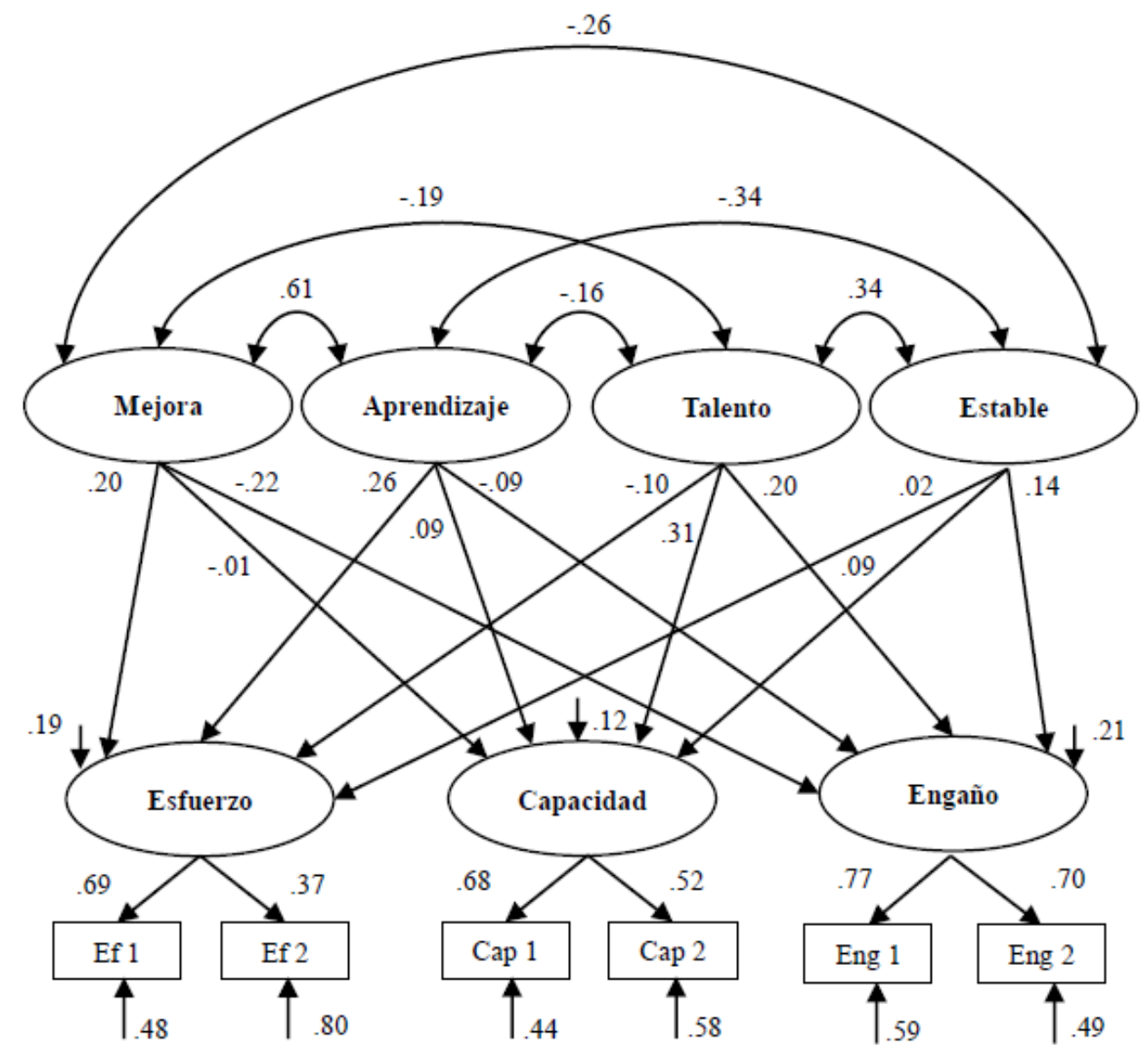

Figura 2. Modelo de regresión estructural mostrando las relaciones entre la percepción de habilidad deportiva y las creencias de éxito. Las creencias de éxito se componen cada una de agrupaciones compuestas de uno o dos ítems. Todos los parámetros están estandarizados y son

estadísticamente significativos en $p<0,05$. Las varianzas explicadas se muestran sobre las flechas pequeñas

\section{DISCUSIÓN}

En relación con las dos primeras hipótesis de este trabajo, los resultados confirman que las creencias implícitas de la habilidad incremental basadas en la mejora y en el aprendizaje predicen positivamente el esfuerzo y negativamente el engaño como creencias de las causas del éxito en el balonmano. Del mismo modo, las dos siguientes hipótesis también son aceptadas en este estudio, es decir, las creencias implícitas de la habilidad como entidad basadas en el talento y la estabilidad predicen positivamente la capacidad y el engaño y negativamente el esfuerzo como causas del éxito en el balonmano. Si bien, no se han hallado estudios a este respecto ni en este deporte ni en la práctica deportiva en general, los resultados vienen a confirmar el postulado de Nicholls (1992) que considera la existencia de una relación entre las creencias implícitas de la habilidad como algo estable o bien adquirido y las diferentes creencias sobre las causas del éxito deportivo (capacidad o esfuerzo). Estos hallazgos son de suma importancia desde el punto de vista 
práctico para el entrenador, dado que señalan que aquellos jugadores con creencias implícitas de habilidad más tendentes a la mejora y a la modificación de su habilidad se esforzarán más por lograr el éxito en su deporte que sus compañeros que creen que la habilidad es estable.

Estos resultados se sitúan en relación con diversas investigaciones (Abraldes et al. 2016; Ruiz-Juan et al., 2010; Usán et al., 2017), las cuales, a pesar de no haber contemplado la totalidad de estas variables, sí que confirman que las creencias implícitas de habilidad incrementales se relacionan como una mayor persistencia en la tarea tanto en los deportes individuales como en los colectivos.

Por otro lado, centrándonos en las creencias implícitas de habilidad como entidad, el talento predijo positivamente la capacidad y el engaño como causas del éxito en el balonmano y de forma negativa el esfuerzo, mientras que las creencias implícitas de la habilidad como estable, predijo positivamente la capacidad, el engaño y el esfuerzo como causas del éxito. Estos resultados pueden situarse en consonancia con Spray et al. (2006) quienes afirman que las creencias implícitas sobre la habilidad pueden ser incrementales y de entidad, percibiendo los deportistas la habilidad deportiva como algo "innato, pero modificable a través del esfuerzo". Por ello, es necesario destacar que posiblemente una creencia implícita sobre la habilidad deportiva como entidad estable no tiene por qué suponer un detraimiento del esfuerzo del deportista, sino que puede complementarse con la creencia implícita incremental.

Finalmente, subrayar que variables como la edad, la modalidad deportiva practicada y el contexto de investigación son elementos a considerar y que pueden explicar, al menos en parte, que los resultados de las diferentes investigaciones analizadas sean tan dispares.

\section{CONCLUSIONES}

Como conclusión, remarcar la existencia de una relación entre las creencias implícitas de los jugadores sobre la habilidad en balonmano con las creencias sobre las causas del éxito en balonmano, encontrando que los jugadores que entienden la habilidad deportiva como incremental se decantan por calificar el éxito deportivo como más dependiente del esfuerzo. De otro modo, los jugadores que conciben la habilidad deportiva como entidad identifican el éxito deportivo dependiente del engaño y de la capacidad personal.

Para futuros trabajos, se podría considerar variables como el género, puesto específico de los jugadores o ranking obtenido en la clasificación, analizando las diferencias existentes en ambas percepciones siguiendo otros estudios recientes (Mosleminezhad, Hemayattalab, \& Fahimi, 2016; Usán et al., 2017). En cuanto a las limitaciones de este trabajo, señalar que el modelo de ecuaciones estructurales 
planteado es el que mejor ajuste presentó, pero se asume que tan solo es uno de los posibles (McDonald \& Ho. 2002).

\section{REFERENCIAS BIBLIOGRÁFICAS}

Abraldes, J. A., Gómez-López, M., Granero-Gallegos, A., \& Rodríguez-Suárez, N. (2013). The goal orientation of the lifesavers and the relationship with the satisfaction and the beliefs about the causes of success in sport. Cultura, Ciencia y Deporte, 22, 59-66. https://doi.org/10.12800/ccd.v8i22.230

Abraldes, J. A., Granero-Gallegos, A., Baena-Extremera, A., Gómez-López, M., \& Rodríguez-Suárez, N. (2016). Orientaciones de meta, satisfacción, creencias de éxito y clima motivacional en nadadores. Revista Internacional de Medicina y Ciencias de la Actividad Física y el Deporte, 16(62), 583-599. https://doi.org/10.15366/rimcafd2016.63.011

Anderson, J. \& Gerbing, D. (1988). Structural equation modeling in practice: A review and recommended two-step approach. Psychological Bulletin, 103(3), 411 423. https://doi.org/10.1037/0033-2909.103.3.411

Bentler, P. (2007). On tests and indices for evaluating structural models. Personality and Individual Differences, 42(5), 825-829. https://doi.org/10.1016/j.paid.2006.09.024

Biddle, S., Wang, C., Chatzisarantis, N., \& Spray, C. M. (2003). Motivation for physical activity in young people: Entity and incremental beliefs about athletic ability. Journal of Sports Science, 21(12), 973-989. https://doi.org/10.1080/02640410310001641377

Bollen, D. A. \& Long, J. S. (1993). Testing structural equation models. Sage: Newbury Parck CA.

Castillo, I., Balaguer, I., \& Duda, J. L. (2002). Las perspectivas de meta de los adolescentes en el contexto deportivo. Psicothema, 14(2), 280-287.

Cervelló, E., Escartí, A., \& Balagué, G. (1999). Relaciones entre la orientación de meta disposicional y la satisfacción con los resultados deportivos, las creencias sobre las causas de éxito en deporte y la diversión con la práctica deportiva. Revista de Psicología del Deporte, 8(1), 7-19.

Cordo-Cabal, L., Gómez-López, M., \& Granero-Gallegos, A. (2019). Relación del clima motivacional generado por el entrenador y las causas del éxito en jugadores de deportes de equipo. Journal of Sport and Health Research, 11(2), 139-150.

Cury, F., Da Fonseca, D., Rufo, M., \& Sarrazin, P. (2002). Perceptions of competence, implicit theory of ability, perception of motivational climate, and achievement goals: a test of the trichotomous conceptualization of endorsement of achievement motivation in the physical education setting. Perceptual and Motor Skills, 95(1), 233-244.

Duda, J. L. \& Nicholls, J. G. (1992). Dimensions of achievement motivation in schoolwork and sport. Journal of Educational Psychology, 84(3), 290-299. https://doi.org/10.1037/0022-0663.84.3.290 
Duda, J. L., Fox, K. R., Biddle, S., \& Armstrong, N. (1992). Children's achievement goals and beliefs about success in sport. British Journal of Educational Psychology, 62(3), 313-323. $\quad$ https://doi.org/10.1111/j.20448279.1992.tb01025.x

Fernández, J., Cecchini, J. A., \& Méndez, A. (2017). Does context, practice or competition affect female athletes' achievement goal dominance, goal pursuit, burnout and motivation? Journal of Human Kinetics, 59(1), 91-105. https://doi.org/10.1515/hukin-2017-0150.

Gómez-López, M., Granero-Gallegos, A., Baena-Extremera, A., \& Abraldes, J. A. (2014). Goal orientation effects on elite handball players motivation and motivational climate. Procedia - Social and Behavioral Sciences, 132, 434440. https://doi.org/10.1016/j.sbspro.2014.04.333

González-Cutre, D., Moreno, J. A., Conte, L., Martínez Galindo, C., Alonso, N., Zomeño, T., \& Marín, L. M. (2007). Predicción de las creencias implícitas de habilidad en deportistas adolescentes a través del clima motivacional percibido en los iguales. En J. Castellano \& O. Usabiaga (Eds.), Investigación en la Actividad Física y el Deporte II (pp. 437-443). Vitoria: Universidad del País Vasco.

Granero-Gallegos, A., Gómez-López, M., Rodríguez-Suárez, N., Abraldes, J. A., Alesi, M., \& Bianco, A. (2017). Importance of the motivational climate in goal, enjoyment, and the causes of success in handball players. Frontiers in Psychology, 8, 2081. https://doi.org/10.3389/fpsyg.2017.02081

Guivernau, M., \& Duda, J. L. (1994). Psychometric properties of a Spanish version of The Task and Ego Orientation in Sport Questionnaire (TEOSQ) and Beliefs about the Causes of Success Inventory. Revista de Psicología del Deporte, 5, 31-51.

Hair, J. F., Black, W. C., Balbin, B. J., \& Anderson, R. E. (2009). Multivariate Data Analysis ( $7^{\text {th }}$ Ed.). New York: Pearson/Prentice Hall.

Hu, L. \& Bentler, P. (1999). Cutoff criteria for fit indexes in covariance structure analysis: Conventional criteria versus new alternatives. Structural Equation Modeling: $\quad A \quad$ Multidisciplinary Journal, 6(1), 1-55. https://doi.org/10.1080/10705519909540118

Jõesaar, H. \& Hein, V. (2011). Psychosocial determinants of young athletes' continued participation over time. Perceptual and Motor Skills, 113(1), 51-66. https://doi.org/10.2466/05.06.13.PMS.113.4.51-66.

King, R. \& Mclnerney, M. (2012). Including social goals in achievement motivation research: Examples from the Philippines. Online Readings in Psychology and Culture, 5(3). 1-24. https://doi.org/10.9707/2307-0919.1104

Kline, R. (2005). Principles and Practice of Structural Equation Modeling (2 ${ }^{\text {nd }}$ Ed.). New York: The Guilford Press.

Laparidis, K., Papaioannou, A., Vretakou, V., \& Morou, A. (2003). Motivational climate, beliefs about the bases of success, and sportsmanship behaviors of profesional basketball athletes. Perceptual and Motor Skills, 96(3 Pt 2), 11411151. 
Li, C. H., Chi, L., Yeh, S. R., Guo, K. B., Ou, C. T., \& Kao, C. C. (2011). Prediction of intrinsic motivation and sports performance using $2 \times 2$ achievement goal framework. Psychological Reports, 108(2), 625-637. https://doi.org/10.2466/05.11.14.PR0.108.2.625-637

Li, W. \& Lee, A. (2004). A review of conceptions of ability and related motivational constructs in achievement motivation. Quest, 56, 439-461. https://doi.org/10.1080/00336297.2004.10491836

Li, W., Lee, A. M., \& Solmon, M. A. (2005). Relationships among dispositional ability conceptions, intrinsic motivation, perceived competence, experience, persistence, and performance. Journal of Teaching in Physical Education, 24(1), 51-65. https://doi.org/10.1123/jtpe.24.1.51

Markland, D. (2007). The golden rule is that there are no golden rules: A commentary on Paul Barrett's recommendations for reporting model fit in structural equation modelling. Personality and Individual Differences, 42(5), 851-858. https://doi.org/10.1016/j.paid.2006.09.023

Marsh, H. (1994). Sport motivation orientations: Beware of jingle-jangle fallacies. Journal of Sport and Exercise Psychology, 16(4), 365-380. https://doi.org/10.1123/jsep.16.4.365

Mascret, N., Falconetti, J. L., \& Cury, F. (2016). Implicit measures of beliefs about sport ability in swimming and basketball. European Journal of Sport Science, 16(3), 358-364. https://doi.org/10.1080/17461391.2015.1080304

McDonald, R. \& Ho, M. (2002). Principles and practice in reporting structural equation analyses. Psychological Methods, 7(1), 64-82. https://doi.org/10.1037/1082-989X.7.1.64

Miles, J. \& Shevlin, M. (2007). A time and a place for incremental fit indices. Personality and Individual Differences, 42(5), 869-874. https://doi.org/10.1016/j.paid.2006.09.022

Moreno, J. A., Cervelló, E. M., Martínez, M. C., \& Moreno, R. (2013). Validación de la Escala de Creencias Implícitas de habilidad (CNAAQ-2) al contexto español. Diferencias según la práctica físico-deportiva. RICYDE. Revista Internacional de Ciencias del Deporte, 9(32), 100-113. https://doi.org/10.5232/ricyde2013.03201

Mosleminezhad, M., Hemayattalab, R., \& Fahimi, H. (2016). Compression of selfconfidence, perception of ability and perceived need of competence between winner and loser teams of Young volleyball players. International Journal of Sport Studies, 6(4), 216-219.

Nicholls, J. G. (1989). The competitive ethos and democratic education. Cambridge: Harvard University Press.

Nicholls, J. G. (1992). The general and the specific in the development and expression of achievement motivation. In G. C. Roberts (Ed.), Motivation in sport and exercise (pp. 31-56). Champaign, IL: Human Kinetics.

Ommundsen, Y. (2001). Students`implicit theories of ability in physical education classes: the influence of the motivational aspects of the learning environment. Learning Environments Research, 4, 139-158. https://doi.org/10.1023/A:1012495615828 
Rev.int.med.cienc.act.fís.deporte - vol. 20 - número 77 - ISSN: 1577-0354

Pérez, E., Medrano, L., \& Rosas, J. S. (2013). El Path Analysis: conceptos básicos y ejemplos de aplicación. Revista Argentina de Ciencias del Comportamiento, $5(1), 52-66$.

Real Decreto 971/2007, de 13 de julio, sobre deportistas de alto nivel y alto rendimiento. Boletín Oficial del Estado, núm. 177, de 25 de julio de 2007, pp. 32240 a

32439 . http://www.csd.gob.es/csd/estaticos/altonivel/legislacion/BOE_Real_Decreto DAN_y_Alto_Rendimiento.pdf

Rodríguez-Ayán, M. \& Ruiz, M. (2008). Atenuación de la asimetría y de la curtosis de las puntuaciones observadas mediante transformaciones de variables: Incidencia sobre la estructura factorial. Psicológica: Revista de Metodología y Psicología Experimental, 29(2), 205-227.

Ruiz-Juan, F., Gómez-López, M., Pappous, A., Alacid, F., \& Flores, G. (2010). Dispositional goal orientation, beliefs about the causes of success and intrinsic satisfaction in young elite paddlers. Journal of Human Kinetics, 26 , 123-136. https://doi.org/10.2478/v10078-010-0056-8

Ruiz-Sánchez, V., Gómez-López, M., \& Granero-Gallegos, A. (2017). Clima motivacional y miedo al fallo en las selecciones juveniles territoriales de balonmano. E-balonmano.com: Revista de Ciencias del Deporte, 13(3), 199206.

Ruiz-Sánchez, V., Gómez-López, M., Granero-Gallegos. A., \& GonzálezHernández, J. (2017). Relación del clima motivacional y miedo al fallo en jugadores de alto rendimiento en balonmano. Cuadernos de Psicología del Deporte, 17(3), 55-64.

Sáenz-López, P., Mateos, J. L., Almagro, B. J., \& Conde, C. (2017). Apoyo a la autonomía, creencias implícitas de habilidad y metas de logro en jugadoras de baloncesto en formación. Cuadernos de Psicología del Deporte, 17(3), 199-206.

Spray, C. M., Wang, C. K. J., Biddle, S., Chatzisarantis, N. L., \& Warburton, V. E. (2006). An experimental test of self-theories of ability in youth sport. Psychology of Sport and Exercise, 7(3), 255-267. https://doi.org/10.1016/j.psychsport.2005.05.001

Usán, P., Salavera, C., Murillo, V., \& Álvarez-Medina, J. (2017). Creencias y percepciones del éxito en futbolistas adolescentes. Diferencias entre categorías deportivas y posición clasificatoria. Retos, 31, 207-211.

White, S., Kavussanu, M., Tank, K., \& Wingate, J. (2004). Perceived parental beliefs about the causes of success in sport: relationship to athletes' achievement goals and personal beliefs. Scandinavian Journal of Medicine and Science in Sports, 14(1), 57-66. https://doi.org/10.1111/j.1600-0838.2003.00314.x

Zamarripa, J., De la Cruz, M. F., Álvarez, O., \& Castillo, I. (2016). Creencias implícitas y orientaciones de meta de jugadoras mexicanas de sóftbol élite. Retos, 30, 184-188.

Número de citas totales / Total references: 47 (100\%) 
Rev.int.med.cienc.act.fís.deporte - vol. 20 - número 77 - ISSN: 1577-0354

Número de citas propias de la revista / Journal's own references: $1(2,32 \%)$

Rev.int.med.cienc.act.fís.deporte - vol. 20 - número 77 - ISSN: 1577-0354 